\title{
Identification of drought responsive Elaeis guineensis WRKY transcription factors with sensitivity to other abiotic stresses and hormone treatments
}

\author{
Fong Chin Lee ${ }^{1,2^{*}}$, Wan Chin Yeap ${ }^{1}$, David Ross Appleton ${ }^{1}$, Chai-Ling Ho² and Harikrishna Kulaveerasingam³
}

\begin{abstract}
Background: The ability of plants to withstand and thrive in an adverse environment is crucial to ensure their survivability and yield performance. The WRKY transcription factors (TFs) have crucial roles in plant growth, development and stress response, particularly drought stress. In oil palm, drought is recognized as one of the major yield limiting factors. However, the roles of WRKYTFs in the drought response of oil palm is unclear.

Results: Herein, we studied the transcriptome of drought treated oil palm leaf and identified 40 differentially expressed genes (DEGs) of WRKYTFs, of which 32 DEGs were upregulated and 8 DEGs were downregulated in response to drought stress in oil palm. They were categorized into Groups I to IV based on the numbers of WRKY domain and the structural difference in the zinc finger domain. Multiple stress- and hormone-responsive cis-regulatory elements were detected in the drought responsive oil palm EgWRKY (Dro-EgWRKY) genes. Fourteen of the 15 selected oil palm WRKY (EgWRKY) genes demonstrated a tissue-specific expression profile except for EgWRKY28 (Group I), which was expressed in all tissues tested. The expression levels of 15 candidate EgWRKYS were upregulated upon salinity and heat treatments, while several genes were also inducible by abscisic acid, methyl jasmonate, salicylic acid and hydrogen peroxide treatments. Members of the Group III WRKYTFs including EgWRKY07, 26, 40, 52, 59, 73 and 81 displayed multiple roles in drought- and salinity-response under the modulation of phytohormones.
\end{abstract}

Conclusions: EgWRKYTFs of oil palm are involved in phytohormones and abiotic stress responses including drought, salinity and heat. EgWRKY07, 26, 59 and 81 from Group III maybe important regulators in modulating responses of different abiotic stresses. Further functional analysis is required to understand the underlying mechanism of WRKY TFs in the regulatory network of drought stress.

Keywords: Oil palm, Drought, Salinity, Heat, Abscisic acid, Salicylic acid, Hydrogen peroxide, Group III WRKY, Reactive oxygen species

\footnotetext{
*Correspondence: lee.fong.chin@simedarbyplantation.com

${ }^{1}$ Sime Darby Plantation Technology Centre Sdn. Bhd, 43400 Serdang,

Selangor, Malaysia

Full list of author information is available at the end of the article
}

\begin{abstract}
Background
WRKY protein is one of the largest transcription factor (TF) family found in the plant kingdom. There are 197 WRKY members in Glycine max [1], 160 members in Triticum aestivum [2] and 145 members in Brassica rapa [3]. WRKY TF is characterized by the presence of a highly conserved WRKY domain comprising of two parts, the DNA-binding heptapeptide WRKYGQK
\end{abstract}


and the zinc finger binding motif which spans about 60 amino acids in length [4]. Other forms of heptapeptide found in the WRKY TF include WRKYGKK, WKKYGQK, WRKYGQR and WRKYGEK [5]. The zinc finger structure can be divided based on the $\mathrm{C} 2 \mathrm{H} 2$ motif (C-X4-5-C-X22-23-H-X1-H) and the C2HC motif (C-X5-7-C-X2-3-H-X1-C) [4]. The C-terminus of WRKY domain has been shown to have a high binding affinity to its cognate cis-acting element, designated as W-box $(\mathrm{C} / \mathrm{T}) \mathrm{TGAC}(\mathrm{T} / \mathrm{C})$ via positively charged $\beta$-strands [4]. As a result, the WRKY proteins are categorized into three groups based on the number of WRKY domains and the zinc finger binding motifs. Group I members have two WRKY domains at both termini whereas Group II and III members have only one WRKY domain. Group I and II members share the same $\mathrm{C} 2 \mathrm{H} 2$-type zinc finger motif while Group III members have the C2HC-type. Furthermore, Group II members can be divided into five subgroups based on their phylogenetic relationships [6].

Being a TF superfamily, WRKY is involved in many biological processes at different stages of the plant life cycle with great emphasis on plant defence response towards biotic and abiotic stresses through the transcription regulation of stress-responsive genes modulated by phytohormones. WRKY TFs are functionally expressed during pollen development $[7,8]$, adventitious root formation [9], flowering [10], leaf senescence [11], callus development [12] and homeostasis of phosphate [13]. Numerous studies have reported the involvement of WRKYS in the response of plants to multiple abiotic stresses such as drought, submergence, heat, cold, salinity and ion toxicity in various plants [14-17], under the influence of phytohormone signals, particularly ABA. In Arabidopsis, AtWRKY46 was upregulated by drought, salinity, SA and hydrogen peroxide $\left(\mathrm{H}_{2} \mathrm{O}_{2}\right)$ treatments [18]. GhWRKY41 [16] from Gossypium hirsutum responded positively to drought and salt stresses in transgenic Nicotiana benthamiana via modulation of reactive oxygen species [19] production in ABA-dependent manner. Extensive studies in T. aestivum disclosed multiple WRKYs involved in different abiotic stresses particularly in drought and salt stresses such as TaWRKY1, TaWRKY33 [17] and TaWRKY46 [20]. TaWRKY46 exhibited an enhanced tolerance to mannitol treatment in transgenic Arabidopsis by increasing the expression of several stress-related genes, namely $\Delta$-1-pyrroline-5-carboxylate synthetase 1 (P5CS1), dehydration-responsive 29B (RD29B), dehydration-response element-binding protein $2 A$ (DREB2A), $A B A$-response element binding factor 3 (ABF3), C repeat/ dehydration-responsive element-binding factor 2 and 3 (CBF2, 3), via ABA-dependent and ABA-independent pathways [20]. In Fragaria vesca, the expression of FvWRKY42 was induced by salt, drought, SA, MeJA and ABA treatments, and overexpression of FvWRKY42 enhanced salt and drought stress tolerance [21].

Oil palm is a highly productive oil crop contributing to approximately $40 \%$ of global vegetable oil demand as food, animal feed and fuel, produced from less than 5.0$5.5 \%$ of the total global oil crop area (approximately 425 Mha) in year 2020 [22]. Oil palm yield is critically affected by environmental factors, particularly drought stress resulting from low rainfall and extended dry seasons caused by climate change, such as the El Niño events. The severe El Niño events in 1997-1998 and 2015-2016 caused declination of palm oil yield [23]. Drought stress caused long term impacts including abnormal frond development, low floral sex ratio leading to yield loss [24], hence, it is imperative to understand the underlying molecular events that cause these responses during drought stress to improve oil palm adaptability and tolerance. Xiao et al. [25] reported 95 members of WRKY TFs in oil palm genome and $17 \mathrm{EgWRKYs}$ upregulated at 2-fold or higher by cold stress based on the transcriptomic data [25]. Out of $17 E g W R K Y s$ genes, six of these cold-responsive $E g W R K Y s$ were also found to be induced by drought and salinity stresses [25]. However, little is known about the repertoire of WRKY transcripts in the transcriptome of drought treated oil palms and the response of these WRKY genes to different phytohormones. We analysed the transcriptomes of oil palm seedlings under drought stress to identify differentially expressed EgWRKY TFs involved in drought stress response. Phylogenetics analysis and gene expression characterization of these genes in response abiotic stresses and phytohormones were also conducted to close the current knowledge gap as well as to evaluate the potential of oil palm WRKY TFs in conferring drought tolerance.

\section{Results}

\section{Identification of Dro-EgWRKY genes}

from the transcriptome of drought-treated oil palms

To elucidate the roles of TFs in drought response, we first identified the TFs among the DEGs identified from the RNA-Seq study on oil palm treated with drought stress in comparison to untreated control oil palm. A total of 6998 DEGs were identified from the RNA-seq analysis; whereby 4175 DEGs were upregulated and 2823 DEGs were downregulated in response to drought stress (Fig. 1A). Approximately 10\% or 675 DEGs among the identified DEGs were TF genes; 389 were upregulated and 286 were downregulated in response to drought stress (Fig. 1B). They were categorized into different TF families, such as AP2-EREBP, bHLH, bZIP, C2H2, MYB, NAC, Orphans, SBP and WRKY which are known as stress-related TF families (Fig. 1C). Among the 40 WRKYs identified, 32 WRKYs were upregulated by 


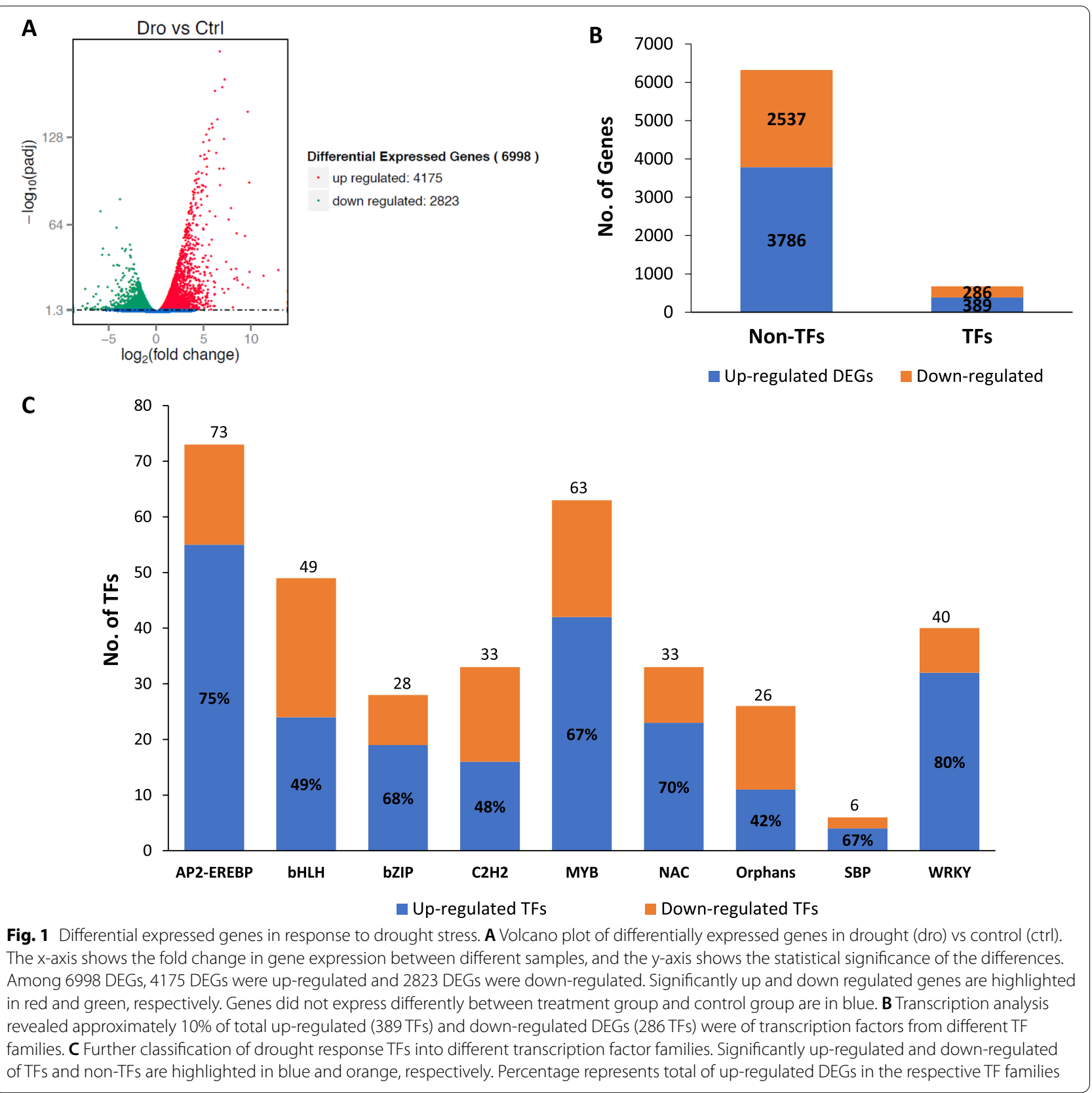

drought stress, representing the highest percentage (80\%) of TF family that was upregulated by drought stress while 8 WRKYs were downregulated (Fig. 1C). WRKY TFs have gained much attention recently in stress response particularly drought stress in many plants [26, 27]. Drought affects oil palm yield, however, limited knowledge is available on the function of WRKYs in drought stress response in oil palm. Thus, 40 differentially expressed WRKY genes were further analysed to understand their roles in abiotic stress response especially drought stress.

\section{Dro-EgWRKY are structurally classified into Group I-IV}

The 40 drought responsive $E g W R K Y$ genes were named and categorized (Table 1) with reference to the oil palm WRKY TFs mentioned in [25]. The chromosomal locations of $3 E g W R K Y$ genes (EgWRKY84, 88 and 94) which were not mapped earlier by Xiao et al. [25] were now successfully mapped to chromosome (CHR) 1, 2 and 15 respectively using the improved version of oil palm genome assembly [19]. EgWRKYO6 has the shortest open reading frame of $528 \mathrm{bp}$ encoding for 176 amino acids while $E g W R K Y 18$ has the longest open reading frame of 


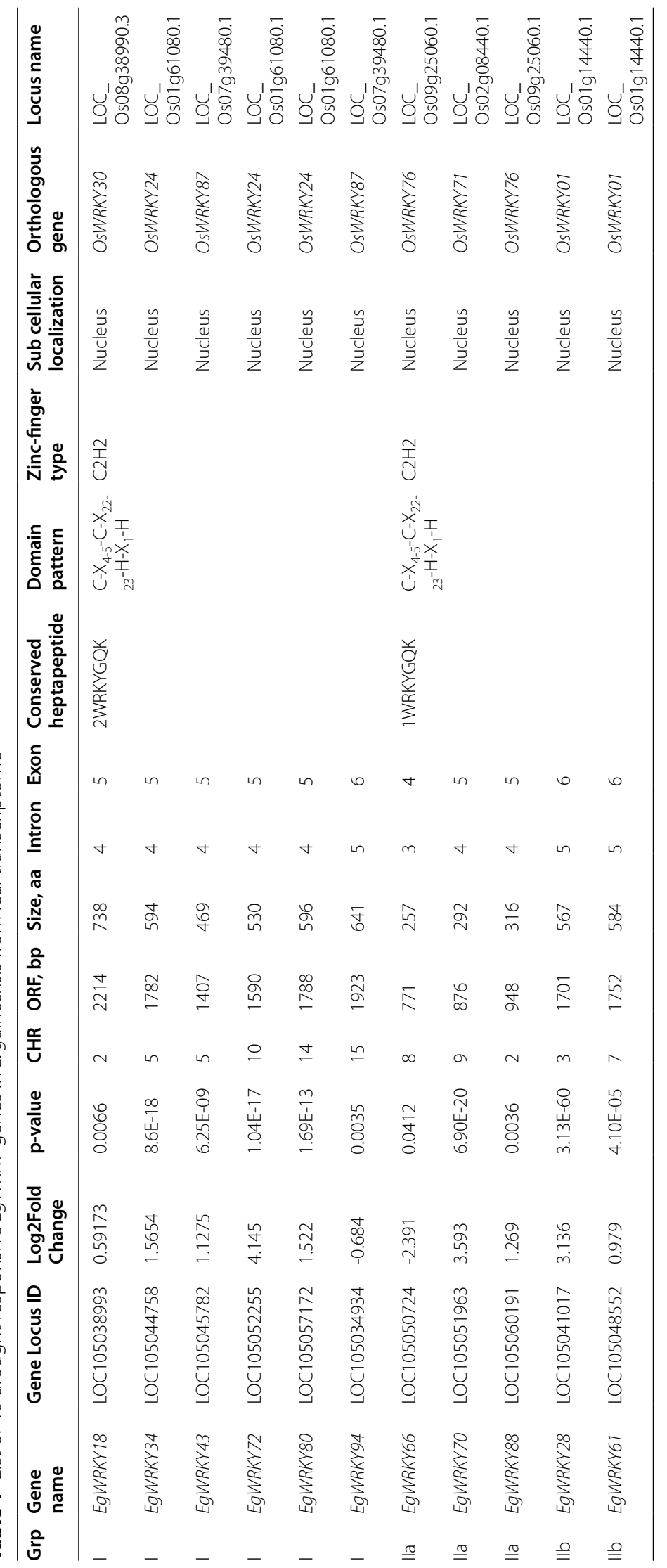




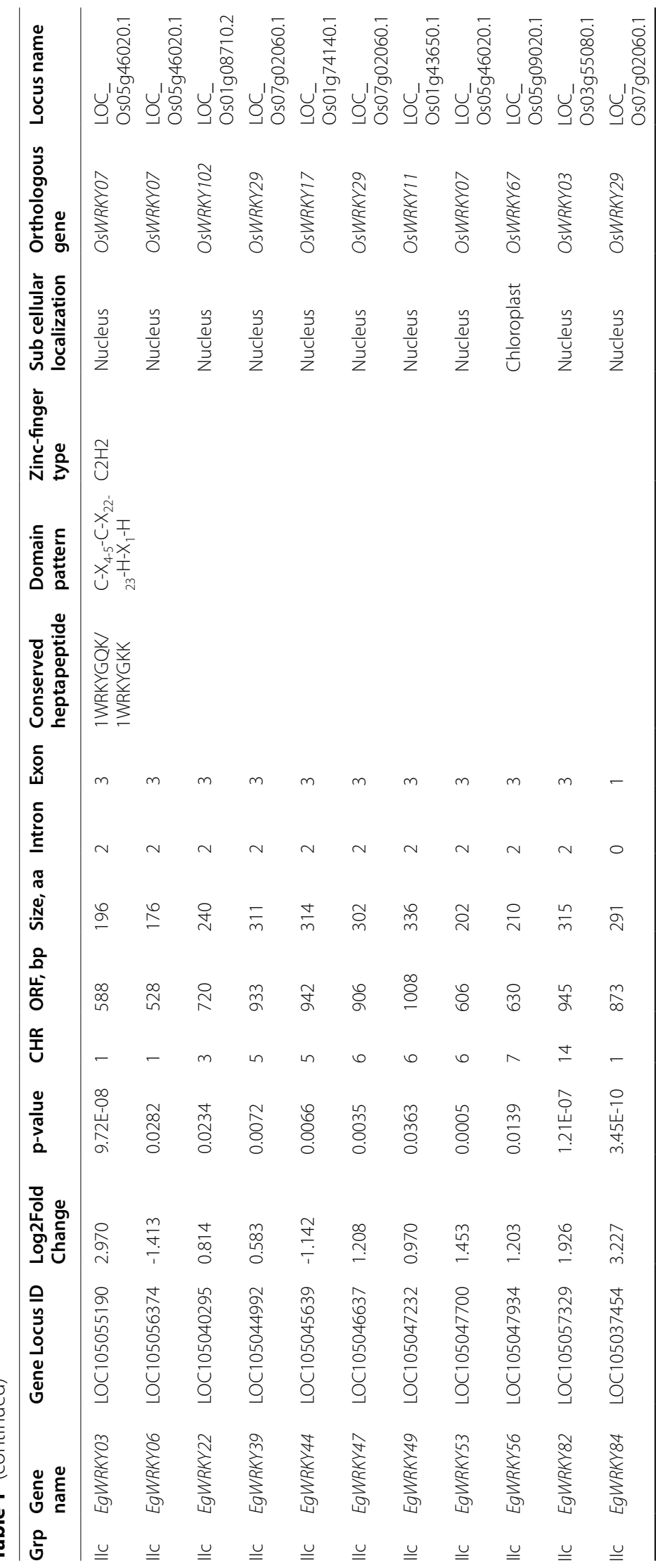




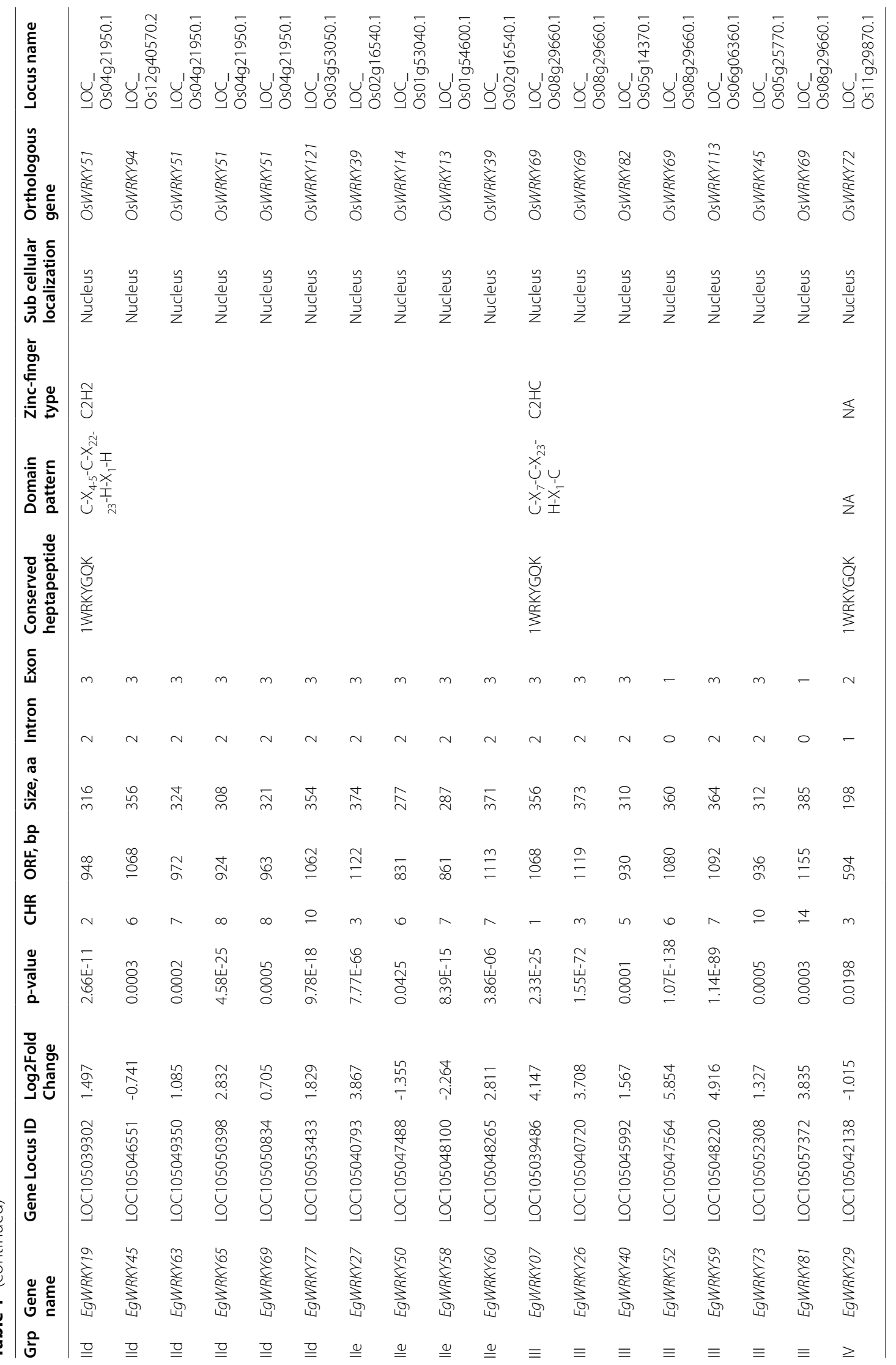


2214 bp encoding 738 amino acids. Most of the EgWRKY genes were predicted to be localized in the nucleus except for EgWRKY56 which was predicted to be localized in the chloroplast. To gain insights into the functions of targeted $\operatorname{EgWRKY}$ genes, we searched for the rice WRKY proteins with the highest identities. Oil palm $E g W R K Y$ genes that have the highest sequence identities to the same rice WRKY gene may display a similar expression pattern in response to drought stress. For instance, EgWRKY19, 63, 65, 69 (that share the highest identities with OsWRKY51), EgWRKY07, 26, 52, 81 (that share the highest identities with OsWRKY69), EgWRKY34, 72, 80 (that share the highest identities with OsWRKY24) and EgWRKY39, 47 and 84 (that share the highest identities with OsWRKY29 gene), showed upregulation by drought stress (Table 1).

Gene name was assigned based on by Xiao et al. [25]. Gene locus was determined based on oil palm reference genome accession number PRJNA192219 deposited in NCBI. Gene's boundaries of exons and introns was determined using the GSDS2.0 (http://gsds.gao-lab.org/). Conserved domains analysis of EgWRKY amino acid sequences were identified using CDD database (http:// www.ncbi.nlm.nih.gov/cdd/). Subcellular localization prediction was conducted using WoLF PSORT (https:// wolfpsort.hgc.jp/). Rice orthologous genes identified using BLASTP search with default parameters and top hit was selected based on the alignment result (http:// rice.plantbiology.msu.edu/analyses_search_blast.shtml).

The 40 EgWRKY genes were classified based on the number of conserved heptapeptide and zinc finger pattern in their protein sequences (Table 1). Among the $40 \mathrm{EgWRKY}$ genes, $6 \mathrm{EgWRKY}$ genes were categorized into Group I, 26 EgWRKY genes belong to Group II, 7 $E g W R K Y$ genes in Group III and only EgWRKY29 were categorized in Group IV. Group II was sub-divided into 5 subgroups according to the unique pattern of the WRKY domain. Group IIc consisted of the highest number of $E g W R K Y$ genes among all groups with 11 members that possess either the common conserved heptapeptide pattern, WRKYGQK or a specific conserved heptapeptide patterns, WRKYGKK. The phylogenetic tree depicts the relationship and structural diversity among the 40 EgWRKY genes (Fig. 2A) in the 8 clades representing the 3 main Groups (I, III and IV) and 5 Subgroups (IIa-e), that are similar with the grouping based on the conserved domains (Fig. 2B). EgWRKY29 protein which does not have the zinc finger motif in the WRKY domain was classified in Group IV. Members in Group I showed closer relationship with the members in Subgroup IIc. Multiple sequence alignment of conserved WRKY domain in the 40 EgWRKY proteins was performed to study the variation among and within the Groups or Subgroups
(Fig. 2B). Two members in Subgroup IIb i.e., EgWRKY28 and 61 were $98.3 \%$ identical to each other. Members within other Subgroups also showed higher sequence identity (more than $80 \%$ ) except for Subgroup IIc while the lowest sequence similarity was observed among the members within Group III (50\%).

We further identified the conserved domains in the EgWRKY amino acid sequences using the CDD database. The complete WRKY domain was observed in 39 EgWRKY proteins except for EgWRKY29 in which lacks the zinc finger motif (Fig. 3A). We noticed the presence of plant zinc cluster domain (40 residues) associated with the WRKY domain in all the members of Subgroup IId. Basic leucine zipper (bZIP) domain (70 residues) involved in DNA-binding and dimerization was found in EgWRKY66 of Subgroup IIa. EgWRKY61 protein contains an uncharacterized conserved domain (COG4372) with unknown function while EgWRKY73 protein has a ligand-dependent nuclear receptor-interacting factor 1 (LRIF) domain. Fifteen motifs specific to EgWRKY proteins were identified (Fig. 3B). Motif 1 which comprised of a heptapeptide WRKYG[Q/K]K was widely distributed in all 40 EgWRKY proteins. We observed that the distribution patterns of the motifs were specific to Group or Subgroups, for instance, motifs 3 and 15 were found only in Group I, motif 5 in Subgroup IId and motif 9 in Subgroup IId.

\section{EgWRKY genes are enriched with light-, stress- and hormone-responsive elements in their promoters}

To identify the cis-regulatory elements corresponding to stress response and hormone signalling, we performed in-silico analysis of cis-regulatory elements in the $2 \mathrm{~kb}$ upstream sequence which covers both the 5'-UTR and promoter of the targeted EgWRKY genes. Light-responsive elements (GT1-motif, G-box and Sp1 element) and stress-responsive elements (dehydrinresponsive element, DRE; F-box; cis-acting element involved in low-temperature responsiveness, LTR) were predicted and found to be significantly abundant in the promoter regions of all the 40 differentially expressed EgWRKY genes (Fig. 4A). The highest number of light responsive elements (27 binding sites) was found in the promoter of EgWRKY18 while the highest number of stress-responsive elements (25 sites) was predicted in the promoters of EgWRKY39 and 03. The stress-responsive elements including MBS (MYB TF binding site involved in drought inducibility), LTR and TC-rich repeats (cis-acting element involved in defence and stress responsiveness) were found in the promoter of EgWRKY84. We also found W box element in the promoter of $27 \operatorname{EgWRKY}$ genes and EgWRKY65 has the highest number i.e. 5 copies of $\mathrm{W}$ 

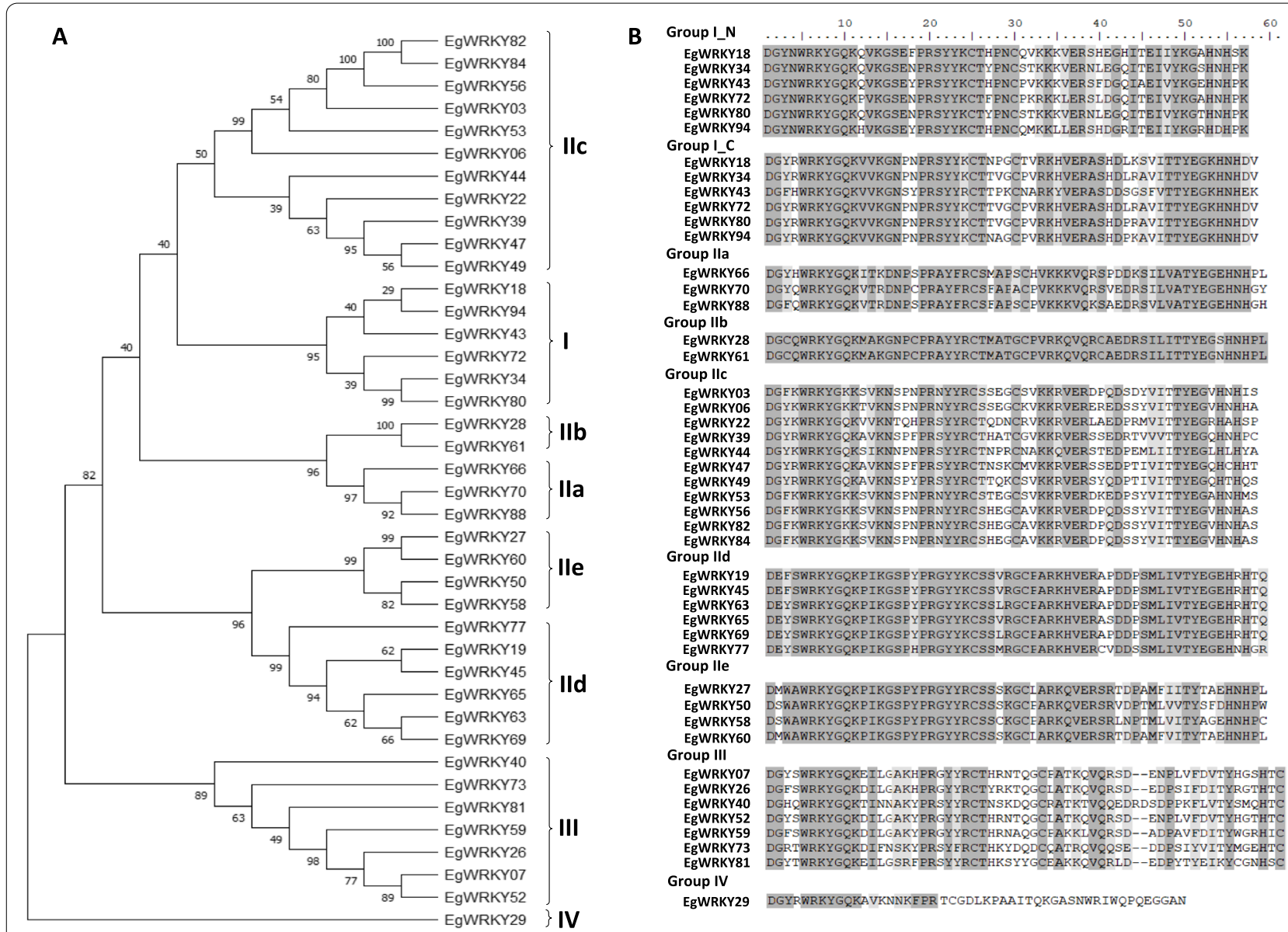

Fig. 2 Phylogenetic analysis and multiple alignment analysis of 40 EgWRKY proteins. A The phylogenetic tree was constructed with MEGA X using UPGMA method based on protein sequences of WRKY domains found in the 40 EgWRKYs which were aligned using Clustal W prior to phylogenetic tree construction using MEGA X based on UPGMA method using Jones-Taylor-Thronton (JTT) substitution model and partial deletion method with 1000 bootstrap value. B Multiple sequence alignment of conserved WRKY domain in 40 EgWRKY proteins was performed using Clustal W programme. Conserved WRKY motif and zinc-finger pattern are indicated within Group or Subgroups with dark grey represents $100 \%$ sequence identity. Groups I_N and I_C indicate the N-terminus and C-terminus of the WRKY domain of Group I EgWRKY protein

box elements, among the $40 \mathrm{EgWRKY}$ genes. The ABAresponsive elements were predicted in the promoter of $36 \mathrm{EgWRKY}$ genes except EgWRKY44, 70, 58 and 5,2 and the highest number (i.e., 16) of binding sites were found in $E g W R K Y 27$. Other hormone-responsive elements like MeJA-responsive (16 in EgWRKY61), salicylic acid-responsive (7 in EgWRKY52) and gibberellin-responsive (4 in EgWRKYO3) were also observed in the promoter of $E g W R K Y$ genes.

\section{Drought responsive DEG EgWRKYs are involved in different growth developmental stages}

To gain insight into the roles of Dro-EgWRKY at different stages of plant growth and development, we examined the expression profile of two randomly selected $E g W R K Y$ members from each group in the mature leaf, young leaf, meristem, root, female inflorescence, zygotic embryo and mesocarp tissues. We noticed a similar expression trend in the members of Group IIb (EgWRKY28 and 61) and Group III (EgWRKYO7 and 26) while the rest of the groups exhibited different tissue specific expression pattern (Fig. 5). Both EgWRKY28 and 61 from Group IIb were highly expressed in the root followed by mature leaf and mesocarp samples. EgWRKYO7 and EgWRKY26 from Group III were expressed predominantly in the vegetative tissues including mature leaf, young leaf, meristem and root. Among the 15 selected $E g W R K Y s, 8$ of them (EgWRKY07, 18, 26, 27, 60, 61, 63 and 65) were expressed in all seven tissues tested while the remaining seven genes had relatively low expression in some tissues. 


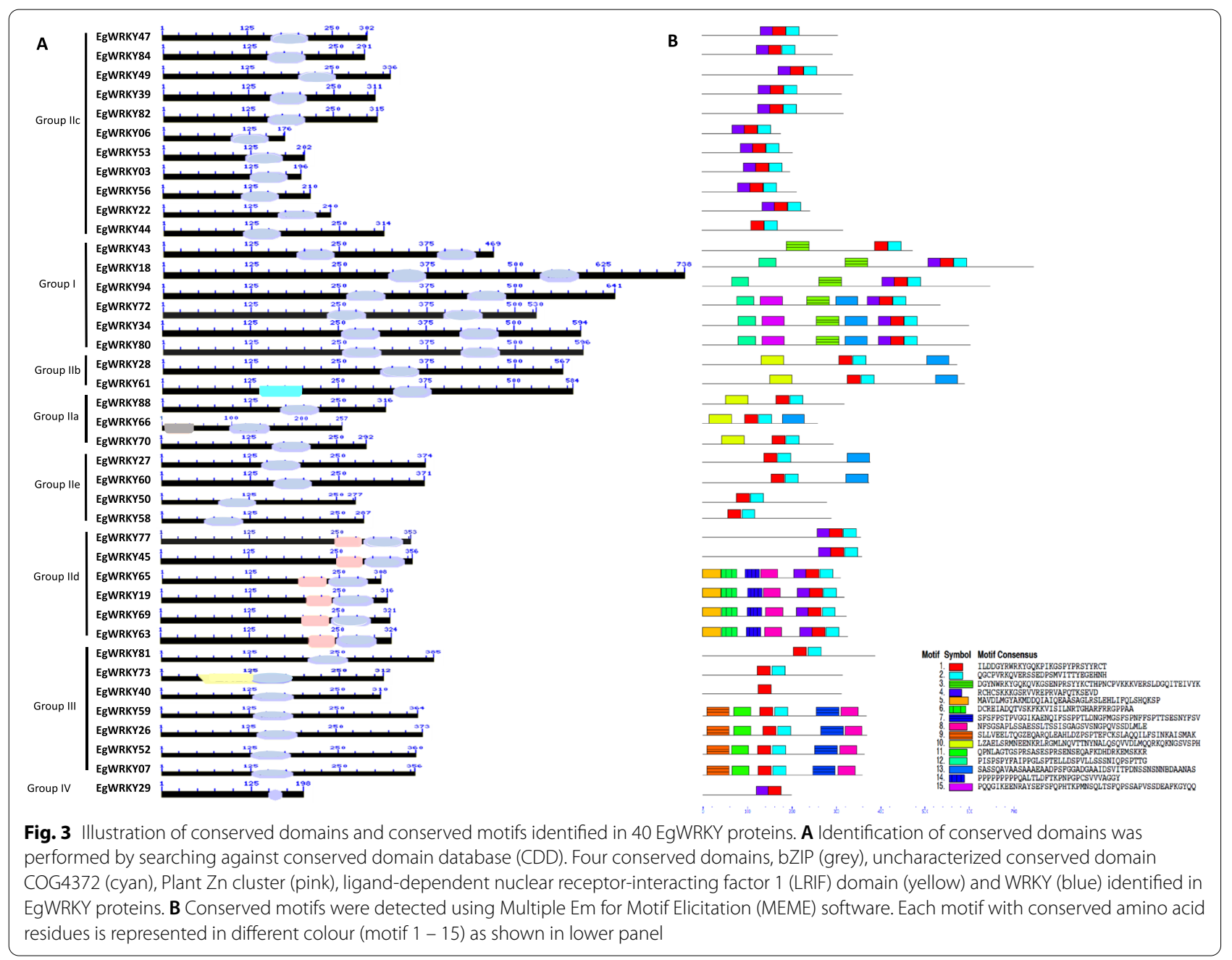

\section{Drought responsive DEG EgWRKYs are responsive to other abiotic stresses, hormones and $\mathrm{H}_{2} \mathrm{O}_{2}$ treatments}

To evaluate the transcript abundance of EgWRKYs in response to different abiotic stresses, we examined the gene expression of 13 upregulated drought responsive EgWRKY with the highest fold change of differential expression identified from RNA-Seq, in cold, drought, flood, heat and salinity treated samples (Fig. 6). Among 13 EgWRKY genes, 5 genes were from Group III, 2 genes each from Subgroup IIc and e while 1 gene each from Group I, Subgroup IIa, b and c. Coincidently, the majority of the highly differentially expressed $\operatorname{EgWRKYs}$, which were expressed higher than 3.7-fold under drought condition were from Group III, hence, the remaining two members $E g W R K Y 40$ and 73 from the same group with lower differential gene expression (1.3-1.5-fold) were also examined. Among the $15 \mathrm{EgWRKY}$ genes analysed, 7 of them (EgWRKY07, 26, 52, 65, 72, 81 and 84) were significantly upregulated by at least 1.3-fold to as high as 6.3-fold by drought, salinity, flood and heat treatments.
However, they were significantly downregulated by cold treatment except for EgWRKYO7 and 84 whereby they were significantly upregulated by cold treatment. Besides, EgWRKY28, 59, 60 and 70 were significantly upregulated by salinity and flood from 1.3-fold to 6.6-fold. Both EgWRKYO3 and 73 were significantly upregulated by salinity treatment but downregulated by cold treatment. No significant differentially gene expression observed in EgWRKY40. Hence, apart from EgWRKY40, all 14 genes being analysed were significantly differentially expressed in all abiotic stress treatments and may be serve as potential stress-responsive candidates for further studies.

Since hormones and $\mathrm{H}_{2} \mathrm{O}_{2}$ are signalling molecules for plant stress responses and we identified various hormones-responsive elements in the promoters of several drought responsive $E g W R K Y s$ TFs, we further conducted hormones (ABA, MeJA and $\mathrm{SA}$ ) and $\mathrm{H}_{2} \mathrm{O}_{2}$ treatments using young leaves to study their effects on the expression of drought responsive EgWRKY genes, harbouring the respective elements in their promoter (Fig. 7). 


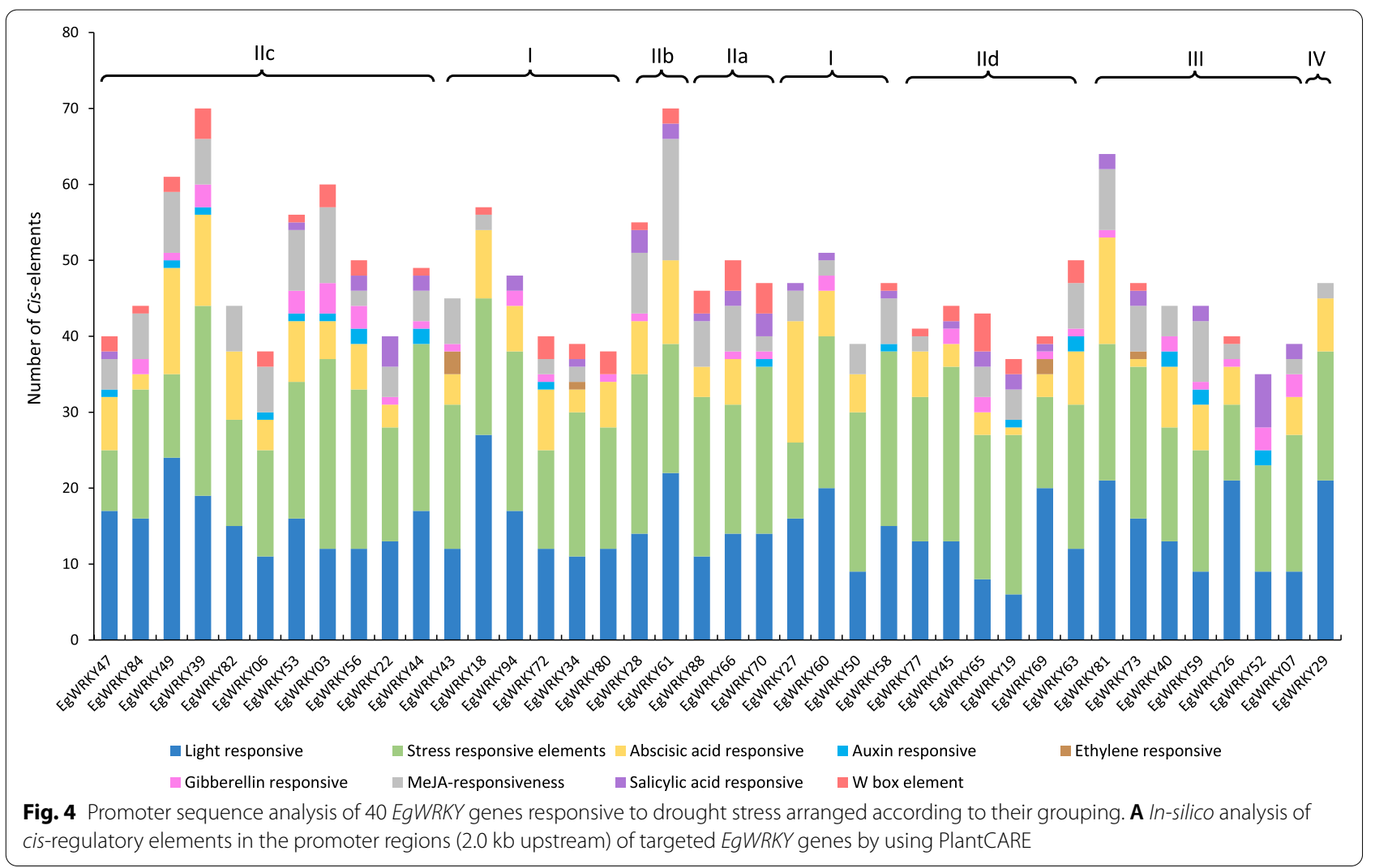

For ABA treatment, EgWRKY18 and 81 with 9 and 14 ABRE elements, respectively were significantly upregulated at 1.5-fold and 1.2-fold, respectively after $1 \mathrm{~h}$ of treatment (Fig. 7A). EgWRKY65 gene with 3 ABRE elements showed significant up-regulation after $30 \mathrm{~min}$ and $1 \mathrm{~h}$ post treatment at 1.1-fold and 1.7-fold, respectively. However, EgWRKY59 was significantly downregulated after $2 \mathrm{~h}$ of treatment. In MeJA treatment, EgWRKY59 was significantly upregulated at 5-fold after 30min of exposure while we observed significant downregulation of EgWRKYO3, 65 and 84 at various time points (Fig. 7B). Interestingly, both EgWRKY70 and 72 were downregulated after $2 \mathrm{~h}$ of exposure, but they were upregulated at $6 \mathrm{~h}$ time point (Fig. 7B). For SA treatment, both EgWRKY59 and 81 were upregulated at different expression level after $30 \mathrm{~min}, 1 \mathrm{~h}$ and $6 \mathrm{~h}$ of exposure (Fig. $7 \mathrm{C}$ ). EgWRKY27, 65 and 70 were significantly upregulated at various time points at different expression level. Then, EgWRKY07 and 27 were downregulated at different expression level after $4 \mathrm{~h}$ of exposure. In $\mathrm{H}_{2} \mathrm{O}_{2}$ treatment, the expression of all six selected $E g W R K Y 52,56,59,65$, 70 and 72 genes were significantly upregulated after 6 $\mathrm{h}$ of exposure and the highest expression was observed in EgWRKY56 and 59 at more than 10-fold higher compared to control (Fig. 7D). Among them, EgWRKY56 was more sensitive to $\mathrm{H}_{2} \mathrm{O}_{2}$ treatment as indicated by early significant positive response after $30 \mathrm{~min}$ of exposure at 1.6-fold and its expression was significantly upregulated at all time points except for $1 \mathrm{~h}$. Besides at $6 \mathrm{~h}$ time point, EgWRKY59, 70 and 72 were also significantly upregulated at different expression level after $4 \mathrm{~h}$ of exposure. Collectively, the findings provide evidence that the selected EgWRKY genes were responsive to different hormonal treatments and highly responsive to $\mathrm{H}_{2} \mathrm{O}_{2}$ treatment at different scale of expression level.

\section{Discussion}

WRKY TFs demonstrate dynamic roles in many aspects of plant life cycle including plant development $[9,10]$, nutrient uptake [13], biotic stress [21, 28] and abiotic stress [18, 27, 29-31]. Extensive studies report that WRKY TFs are potent regulators in conferring drought tolerance in plants [26, 29, 32-37], modulated by phytohormones especially ABA [35]. Oil palm yield is severely affected by drought stress resulting in low flower sex ratio and early fruit bunch abortion [24] which cause huge economic losses. Nevertheless, little is known about the functions of WRKY TFs in drought response in oil palm. Hence, it is crucial to identify $\operatorname{EgWRKY}$ TFs that may improve drought tolerance in oil palm.

In this study, we identified 40 differentially expressed EgWRKY TFs responsive to drought stress in oil palm leaf 

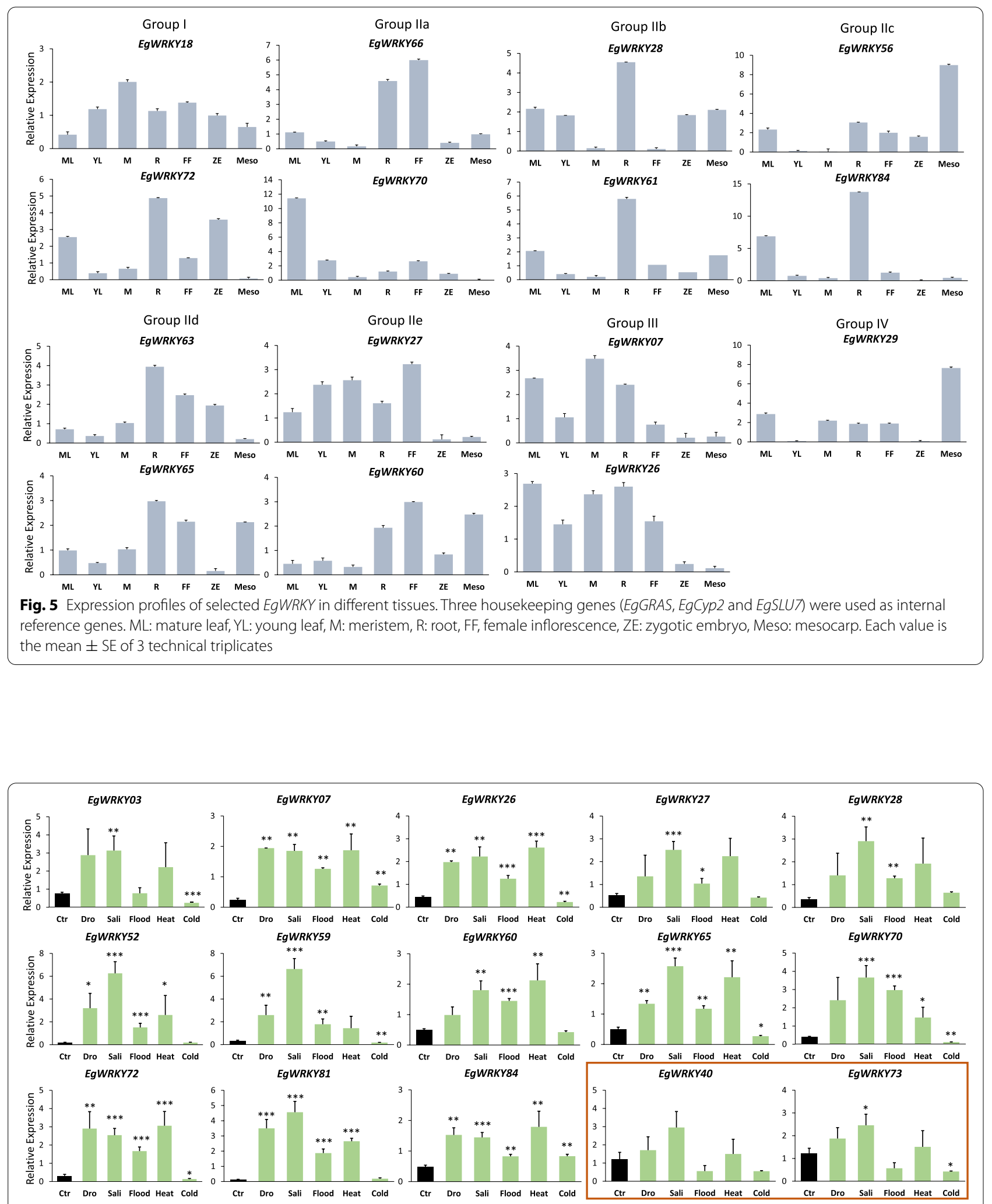

Fig. 6 Expression profiles of selected EgWRKY under different abiotic stress treatments (green) and control (black). Additional candidates genes from Group III that were analyzed are boxed in brown. Three housekeeping genes (EgGRAS, EgCyp2 and EgSLU7) were used as internal reference genes. Each value is the mean \pm SE of 3 independent biological replicates. Asterisks indicate significant differences between each treatment point and controls $\left({ }^{*} P<0.05,{ }^{* *} P<0.01,{ }^{* * *} P \leq 0.001\right.$, Student's t-tests) 


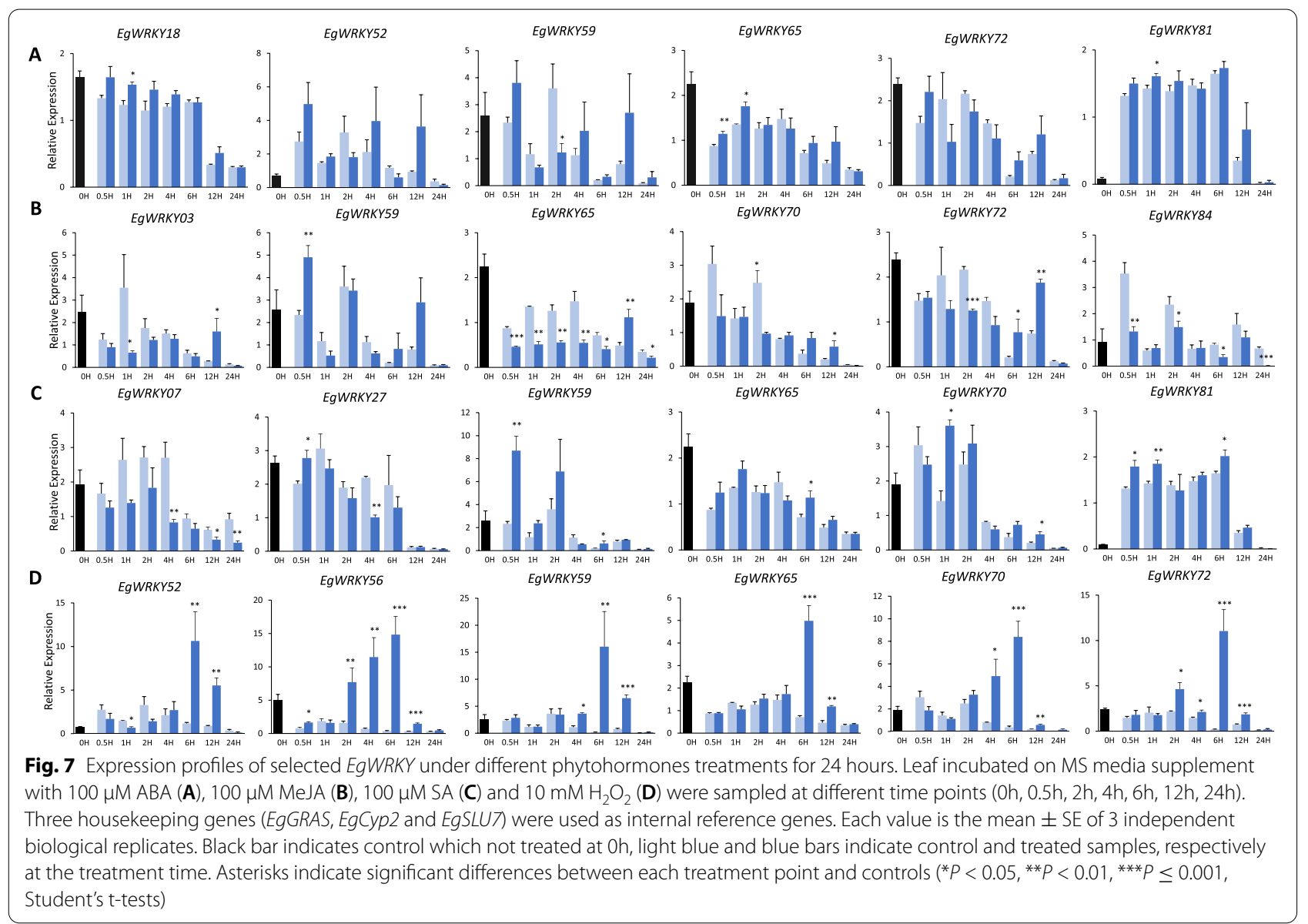

using RNA-seq. We further evaluated their response to different abiotic stresses and phytohormone treatments. Our RNA-seq results revealed that the WRKY TFs were among the TFs that were responsive to drought stress in oil palm with $80 \%$ of them upregulated by drought stress, followed by TFs in the AP2-EREBP, NAC and bZIP families. Further characterization of the EgWRKY candidates in this study will shed light on their possible roles in drought response in oil palm.

A total of 95 WRKY TFs were identified in the oil palm genome by Xiao et al. [25]. They were named according to the chromosomal location and classified into 8 groups based on their phylogenetic relationship [25], instead of following the general classification of WRKY TFs into 3 main Groups I, II and III, with 5 Subgroups in Group II (IIa to IIe) [4]. In our study, we categorized 40 drought responsive EgWRKY DEGs into 4 groups (Groups I, IIa-e, III and IV) according to the general classification method to ease the comparison with WRKY TFs from other plants. Phylogenetic analysis revealed the close relationship of EgWRKY members in Group I and Group II that are represented by 4 clades. Notably, EgWRKY29 was categorized into Group IV due to the absence of an intact zinc finger motif in its WRKY domain, which may be due to a deletion event. In concordance, the presence of Group IV WRKY TFs which lack an intact zinc-finger motif in the WRKY domain is also reported in plants such as Pennisetum glaucum [30] and Ammopiptanthus nanus [38]. We have successfully assigned these 40 drought responsive EgWRKYs to oil palm CHR including EgWRKY84, 88 and 94 genes that could not be mapped to the oil palm reference genome by Xiao et al. [25]. They were now successfully mapped to CHR1, 2 and 15, respectively; using the improved genome assembly [19]. To ease future referencing, we maintained the nomenclature of EgWRKY84, 88 and 94 regardless of their chromosomal locations. It is worth mentioning that the Dro-WRKY TFs were mainly found on CHR3, 5, 6 and 7 which constituted $50 \%$ of the total Dro-WRKY TFs. CHR7 harbours 6 WRKYs of which 5 were upregulated by drought among 9 WRKYs located in CHR7. Hence, this information serves as a good reference for the subsequent effort in identifying drought hotspots in oil palm genome through mapping of the drought responsive DEGs and drought responsive markers discovered in oil palm [39]. 
To understand the functions of the Dro-EgWRKYs, we first profiled their tissue-specific gene expression, subsequently their response to various abiotic stress and phytohormone treatments. WRKY TFs are involved in different developmental stages of plants such as adventitious root formation [9], leaf senescence and flowering [10]. Here, we noticed that EgWRKY70 and 84 were preferentially expressed in mature leaf, both EgWRK29 and 56 were predominantly expressed in mesocarp while EgWRKY61 and 84 were detected abundantly in root tissue, suggesting their possible involvement in different stages of oil palm growth and development. This tissue-specific expression profile is not associated with their grouping as there was no specific expression pattern observed among the members from the same group. However, it must be further validated by analysing more members of same group instead of only 2 members from each group. During the onset of drought stress, root is the first organ that responses to water stress signal while leaf responds to drought stress through stomatal closure [40]. Both EgWRKY61 (Group IIb) and 84 (Group IIc) were highly expressed in the mature leaf and root tissues, indicating their possible roles in responding to water stress signal in both organs during drought stress. WRKY TFs were reported to regulate transcription of stress related genes that respond to multiple abiotic stresses in particular drought and salinity as both shared similar signal transduction pathway [41]. In Arabidopsis, AtWRKY46 was upregulated by drought, salinity, SA and $\mathrm{H}_{2} \mathrm{O}_{2}$ treatments [18]. GhWRKY41 [16] from G. hirsutum were upregulated by drought and salt stress in the transgenic $N$. benthamiana by removing ROS to better adjust the osmotic stress in a ABA-dependent manner [19]. In oil palm, 13 selected EgWRKYs were found to be significantly expressed in samples treated with different abiotic stresses, especially EgWRKY26, 65, 72 and 81 that exhibited a significant increase in expression levels in response to drought, heat and salinity. These 4 candidates were also identified as DEGs in oil palm under salinity and heat stresses (unpublished data). Collectively, these results suggest that the drought responsive $\operatorname{EgWRKY}$ TFs may be involved in the regulation of multiple abiotic stress responses, possibly sharing the same mechanism in responding to environmental stimuli to induce stress related genes.

Much attention has been channelled to study the functions of Group III members of WRKY TFs due to their involvement in multiple processes from plant development to stress signalling response [42-44]. The Group III members are distinguishable from other Group members by the presence of zinc finger type $\mathrm{C} 2 \mathrm{HC}$ instead of $\mathrm{C} 2 \mathrm{H} 2$. Herein, we validated that the expression levels of all 7 members of Group III WRKYs including
EgWRKY07, 26, 40, 52, 59, 73 and 81 were upregulated by drought stress in oil palm seedlings. We also showed that they were upregulated by other abiotic stresses including cold, flood, heat and salinity. In G. hirsutum, the Group III WRKY members including GhWRKY31, 59 and 102 were also involved in fibre development and leaf senescence apart from abiotic stress response [43]. Furthermore, many Group III members of WRKY TFs have also been reported to be involved in biotic stress response. For instance, six members of the Group III WRKY TFs from Solanum lycopersicum were involved in the tomato yellow leaf curly virus (TYLCV) defence signalling pathway [45], and ScWRKY5, a Group III WRKY gene from sugarcane was upregulated by fungal infection, drought, salinity and hormonal stresses [44]. We observed a relatively higher gene expression levels of two Group III WRKY members (EgWRKYO7 and 26) in the oil palm vegetative tissues including mature leaf, young leaf and root. This suggests that some Group III drought responsive $E g W R K Y$ TFs may play multiple roles in abiotic stress response, growth and development in oil palm. In addition, further analysis on the sequences of EgWRKY TFs in oil palm revealed a total of $13 E g W R K Y$ TFs from Group III (unpublished data). Hence, further investigation on the remaining 6 members of Group III EgWRKY TFs may provide a clear indication of the functions of Group III EgWRKY TFs in oil palm.

In plants, phytohormones play crucial roles in controlling physiological responses to environmental stimuli especially ABA, SA, MeJA and ET via transcriptional modulation of transcription factor genes and stress responsive genes $[15,36,46]$. ABA is known as a stress hormone as it positively regulates plant responses to different environmental stresses particularly drought stress by triggering stomatal closure to reduce the transpirational water loss in leaf during water scarcity [47]. Besides, both SA and MeJA are also involved in attuning plant responses to different abiotic stresses through interaction with ABA, mediated by TFs including MYC, NAC and WRKY [47, 48]. Studies have shown that WRKY TFs regulate plant responses to different stresses under the influence of phytohormones including ABA, SA, MeJA and ET [49]. This subsequently leads to the activation of stress-responsive genes to confer tolerance to bacteria pathogen [50], fungal pathogen [21] and to abiotic stresses like drought, salinity, heat and cold $[16,17]$. In the current study, we examined the effects of ABA, SA and MeJA on the gene expression levels of drought responsive $E g W R K Y$ genes harbouring the corresponding cis-elements in their promoter. We provide evidence that both EgWRKY65 and 81 were upregulated by drought, heat, salinity, flood stresses and hormonal treatments which included ABA and SA at certain time 
points. These results suggest a crosstalk between ABA and SA in the signalling transduction to trigger response against different abiotic stresses which is in agreement with $Z m W R K Y 40$ from maize [51]. Interestingly, EgWRKY59 gene (a Group III member) was significantly upregulated by drought, heat, flood and salinity stresses, was also found to be significantly upregulated by three phytohormones including $\mathrm{ABA}, \mathrm{MeJA}$ and SA at certain time points, suggesting the potential involvement of EgWRY59 in the regulatory network of three abiotic stresses via crosstalks of three phytohormones. Hence, EgWRKY59 is one of the potential candidates for further characterization as abiotic stress markers in oil palm.

During the onset of abiotic stress, excessive $\mathrm{ROS}\left(\mathrm{H}_{2} \mathrm{O}_{2}\right.$, superoxide radicals, hydroxyl radical) are generated that cause oxidative stress to plants and subsequently inhibit normal growth and reproduction in plants [52]. Plants develop different defence systems to counter oxidative stress through the activation of ROS scavenging enzymes (superoxide dismutase and peroxidases), regulation of the downstream stress response genes and accumulation of osmoprotectants such as proline, glycine and trehalose [53, 54]. Here, we examined the expression levels of drought responsive $E g W R K Y s$ in response to high oxidative stress induced by $\mathrm{H}_{2} \mathrm{O}_{2}$ treatment. All 6 EgWRKYs (52, 56, 59, 65, 70 and 72) were highly sensitive to $\mathrm{H}_{2} \mathrm{O}_{2}$ treatment with upregulation of transcript levels after $6 \mathrm{~h}$ of treatment. This was also observed in the expression profiles of Aquilaria. sinensis AsWRKY genes that were upregulated upon $\mathrm{H}_{2} \mathrm{O}_{2}$ treatment, in particular AsWRKY25 that increased 100-fold in gene expression after $12 \mathrm{~h}$ of treatment [55]. In addition, many studies reported the involvement of WRKY TFs in reducing oxidative stress by controlling the stomatal closure mediated by ABA and SA $[15,36,56]$. Here, we found that EgWRKY59 and EgWRKY65 were upregulated by $\mathrm{ABA}$ and SA treatments, and they were also significantly upregulated by drought, heat, flood and salinity treatments in oil palm. These findings collectively suggest a crosstalk between $\mathrm{H}_{2} \mathrm{O}_{2}$ and phytohormones (ABA, $\mathrm{SA})$ in regulating the gene expression of EgWRKY59 and EgWRKY65 in adaptation to the increase in ROS level, probably through the transcription regulation of genes encoding ROS scavenger. Further characterization is required to provide more evidence to support this observation.

\section{Conclusion}

A total of 40 DEGs encoding WRKY TFs were identified from the transcriptome of drought treated leaf of oil palm. All 32 upregulated Dro-EgWRKY genes have preferential expression in different tissues, and exhibited different response to abiotic stresses, phytohormones and $\mathrm{H}_{2} \mathrm{O}_{2}$ treatments. EgWRKY59 and EgWRKY65 may share similar regulatory mechanism involving $\mathrm{ABA}-\mathrm{SA}$ - and ROS-mediated signalling during drought and other abiotic stresses and are potential candidate genes for conferring higher tolerance to these types of stress. EgWRKY07, 26, 59 and EgWRKY81 from Group III may involve in the regulation of different abiotic stress responses. Further functional studies of these candidate genes are required to evaluate their potential as drought biomarker to screen for oil palm with better drought tolerance and as candidate genes for genetic improvement purpose.

\section{Methods \& materials}

\section{Plant materials, growth conditions and treatments}

Mature leaf, young leaf, female inflorescence, zygotic embryo and mesocarp were sampled from 15-year-old commercial DxP (Deli Dura x Pisifera) palm. Meanwhile, meristem and root were sampled from 1-year-old DxP (Deli Dura x Pisifera) seedling. For different abiotic stress treatments, 6-month-old oil palm Dura (Deli Dura) seedlings planted in polybags filled with top soil were acclimatized in greenhouse environment for 1 month at 28 ${ }^{\circ} \mathrm{C}$ prior to exposure to abiotic stress treatments for two weeks at $28{ }^{\circ} \mathrm{C}$ except for the cold and heat treatments. Six biological replicates of oil palm seedlings were used for each stress treatment. Three independent oil palm seedlings and three untreated oil palm seedlings (controls) were randomly selected for RNA-Seq study. For control, seedlings were watered daily with $200 \mathrm{~mL}$ of tap water. For seedlings in the drought treatment, no watering was conducted for two weeks. For flood treatment, the water level was maintained one inch above the soil level. In salinity treatment, seedlings were irrigated with $200 \mathrm{~mL}$ of $200 \mathrm{mM} \mathrm{NaCl}$ daily. Seedlings were incubated at $15{ }^{\circ} \mathrm{C}$ and $35{ }^{\circ} \mathrm{C}$ while watering remain the same as control seedling for cold and heat treatment, respectively. Hormonal and $\mathrm{H}_{2} \mathrm{O}_{2}$ treatments were conducted on the young leaf pieces of $2 \times 2 \mathrm{~cm}$ dimension incubated on MS agar plate supplemented with $100 \mu \mathrm{M} \mathrm{ABA}, 100 \mu \mathrm{M}$ MeJA, $100 \mu \mathrm{M} \mathrm{SA}, 10 \mathrm{mM} \mathrm{H}_{2} \mathrm{O}_{2}$ for a total incubation period of $6 \mathrm{~h}$. Four leaf pieces were sampled at 0, 0.5, 2, 4 6,12 and $24 \mathrm{~h}$.

\section{RNA-Seq analysis}

Total RNA of oil palm leaves was isolated from 3 control and 3 drought treated oil palm seedlings sharing the same parents using MN Nucleospin RNA Plant Kit (Macherey-Nagel, Germany) according to the manufacturer's instructions. The quality and concentration of total RNA samples were evaluated using high sensitivity Bioanalyzer chip (Agilent Technologies, USA) prior to library preparation. Sequencing and bioinformatic analysis were 
carried out using Illumina HiSeq2000 (Novogene Bioinformatics Technology, China). After removing adaptor and low quality reads, clean reads were mapped onto oil palm reference genome accession number PRJNA192219 deposited in NCBI, using TopHat2 algorithm with a maximum mismatch set at 2 [57]. Gene expression level was quantified using fragments per kilobase of transcript over million mapped reads (FPKM) method and analysed using HTSeq software [58]. Subsequent identification of DEGs was performed using DESeq software with a corrected $p$-values $<0.05$ [59]. TF analysis on the DEGs was conducted using iTAK program V1.2 according to default parameters [60].

\section{Sequence analysis}

Gene and protein sequences of the Dro-EgWRKY candidates identified from RNA-Seq were retrieved from National Center for Biotechnology Information (NCBI) and matched with the sequences reported by Xiao et al. [25] by using BLAST-N and BLAST-P tools at default setting. Protein sequences of WRKY domain found in the EgWRKYs were aligned using Clustal W prior to phylogenetic tree construction using MEGA X based on UPGMA method using Jones-Taylor-Thronton (JTT) substitution model and partial deletion method with 1000 bootstrap value. . Conserved domains in EgWRKY amino acid sequences were identified using CDD database (http://www.ncbi.nlm.nih.gov/cdd/). The conserved motifs in the full length EgWRKY proteins were analysed using the Multiple Em for Motif Elicitation (MEME) program (https://meme-suite.org/meme/tools/meme). MEME motifs are represented by position-dependent letter-probability matrices which describe the probability of possible letter at each position in the pattern. The maximum number of motifs was set at 15 , the maximum motif length was set at 60 amino acids, the optimum motif width was restricted at 6 to 300 residues, and the other default parameters were used. Subcellular localization prediction was conducted using WoLF PSORT (https://wolfpsort.hgc.jp/). Identification of rice ortholog genes was performed using BLASTP search with default parameters and top hit was selected based on the alignment result (http://rice.plantbiology.msu.edu/analyses_ search_blast.shtml). Identification of cis-regulatory elements in the putative 5'-UTR and promoter regions of the targeted EgWRKY genes was conducted on the 2 $\mathrm{kb}$ upstream sequence from the start codon of genomic sequence using PlantCARE (http://bioinformatics.psb. ugent.be/webtools/plantcare/html/).

\section{Quantitative RT-PCR (qRT-PCR) analysis of transcripts}

The first-strand cDNA was synthesized from total RNA of oil palm tissue using Maxima First Strand cDNA
Synthesis Kit (Thermo Scientific, USA) and quantified using StepOne Plus (Applied Biosystems, USA) and Fast SYBR Green Master Mix (Applied Biosystems, USA) according to the manufacturer's instructions. Dissociation curves were generated to verify the amplification specificity. Independent qRT-PCR runs were conducted in technical triplicates for different tissues and both biological and technical triplicates for abiotic stress, hormonal and $\mathrm{H}_{2} \mathrm{O}_{2}$ treatments and the calibrated normalized relative quantity (CNRQ) values of the transcripts were calculated using delta-delta Ct method [61]. Expression of target genes in oil palm mesocarp were normalized to Gibberellin-responsive protein 2 (EgGRAS), cyclophilin 2 (EgCyp2) and Pre-mRNA splicing factor SLU7 (EgSLU7) [62]. Student's t-test was conducted using the $\log _{2}$ value of relative expression level to evaluate the statistical significance in the differences observed in target gene expression between the control and treated samples.

\section{Abbreviations}

ABA: Abscisic acid; ABF3: ABA-response element binding factor 3; bZIP: Basic leucine zipper; CHR: Chromosome; DEGs: Differentially expressed genes; Dro-EgWRKY: Drought responsive oil palm WRKY; ET: Ethylene; FPKM: Fragments per kilobase of transcript over million mapped reads; $\mathrm{H}_{2} \mathrm{O}_{2}$ : Hydrogen peroxide; MeJA: Methyl jasmonate; : Oil palm WRKY; : EgWRKY; Oil palm WRKY: EgWRKY; qRT-PCR: Quantitative RT-PCR; SA: Salicylic acid; TYLCV: Tomato yellow leaf curly virus; TFs: Transcription factors.

\section{Supplementary Information}

The online version contains supplementary material available at https://doi. org/10.1186/s12864-022-08378-y.

Additional file 1: Table S1. Expression data of differential expressed genes (DEGs) encode for APE-EREBP transcription factors. Table S2. Expression data of differential expressed genes (DEGs) encode for bHLH transcription factors. Table S3. Expression data of differential expressed genes (DEGs) encode for bZIP transcription factors. Table S4. Expression data of differential expressed genes (DEGs) encode for $\mathrm{C} 2 \mathrm{H} 2$ transcription factors. Table S5. Expression data of differential expressed genes (DEGs) encode for MYB transcription factors. Table S6. Expression data of differential expressed genes (DEGs) encode for NAC transcription factors. Table S7. Expression data of differential expressed genes (DEGs) encode for Orphans transcription factors. Table S8. Expression data of differential expressed genes (DEGs) encode for SBP transcription factors.

Additional file 2: Table S9. qRT-PCR primers

Additional file 3. Promoter sequences of 40 Dro-EgWRKY genes used in the promoter sequence analysis (Fig. 4) .

\section{Acknowledgements}

The authors thank Sime Darby Research, Banting, Selangor, Malaysia for the oil palm samples, Dilip Kumar, Norkhairunnisa Che Mohd Khan and Norfadzilah Jamalludin for assistance and support. The authors also thank the handling editors and reviewers for the constructive feedback.

\section{Authors' contributions}

All authors conceived the experiments. FCL and WCY conducted the experiments and analyzed the data. FCL wrote the manuscript and all authors reviewed and edited the manuscript. WCY, DRA and HK involved in funding acquisition. All authors read and approved the final manuscript. 


\section{Funding}

This research was fully supported by internal grants from Sime Darby Plantation, Malaysia.

\section{Availability of data and materials}

The reference genome assembly used for data analysis was obtained from National Center for Biotechnology Information (NCBI) BioProject PRJNA192219. The raw transcriptome data generated and analysed in this study deposited in SRA of the NCBI under accession number PRJNA775831. All data used for the phylogenetic tree analysis can be accessed from Treebase at http://purl.org/phylo/treebase/phylows/study/TB2:S29059. The datasets analysed during this study are included in this published article and its supplementary information files.

\section{Declarations}

\section{Ethics approval and consent to participate}

Plant material used in the study complies with relevant institutional, national, and international guidelines and legislation. Plant material used is not wild species and also permission for the plant material is not required.

\section{Consent for publication}

Not applicable.

\section{Competing interests}

The authors declare that they have no competing interests.

\section{Author details}

${ }^{1}$ Sime Darby Plantation Technology Centre Sdn. Bhd, 43400 Serdang, Selangor, Malaysia. ${ }^{2}$ Department of Cell and Molecular Biology, Faculty of Biotechnology and Biomolecular Sciences, Universiti Putra Malaysia (UPM), 43400 Serdang, Selangor, Malaysia. ${ }^{3}$ Sime Darby Research Sdn Bhd, R\&D Centre, 42700 Banting, Selangor, Malaysia.

Received: 14 September 2021 Accepted: 8 February 2022 Published online: 26 February 2022

\section{References}

1. Rushton PJ, Somssich IE, Ringler P, Shen QJ. WRKY transcription factors. Trends Plant Sci. 2010;15(5):247-58.

2. Okay S, Derelli E, Unver T. Transcriptome-wide identification of bread wheat WRKY transcription factors in response to drought stress. Mol Gen Genomics. 2014;289(5):765-81.

3. Kayum MA, Jung HJ, Park Jl, Ahmed NU, Saha G, Yang TJ, et al. Identification and expression analysis of WRKY family genes under biotic and abiotic stresses in Brassica rapa. Mol Gen Genomics. 2015;290(1):79-95.

4. Eulgem T, Rushton PJ, Robatzek S, Somssich IE. The WRKY superfamily of plant transcription factors. Trends Plant Sci. 2000:5(5):199-206.

5. Zhang Y, Wang L. The WRKY transcription factor superfamily: its origin in eukaryotes and expansion in plants. BMC Evol Biol. 2005;5(1):19-12.

6. Liu A, Liu C, Lei H, Wang Z, Zhang M, Yan X, et al. Phylogenetic analysis and transcriptional profiling of WRKY genes in sunflower (Helianthus annuus L.): genetic diversity and their responses to different biotic and abiotic stresses. Ind Crop Prod. 2020;148:112268.

7. Wang Y, Li Y, He SP, Gao Y, Wang NN, Lu R, et al. A cotton (Gossypium hirsutum) WRKY transcription factor (GhWRKY22) participates in regulating anther/pollen development. Plant Physiol Biochem. 2019;141:231-9.

8. Lei R, Li X, Ma Z, LvY, Hu Y, Yu D. Arabidopsis WRKY2 and WRKY34 transcription factors interact with VQ20 protein to modulate pollen development and function. Plant J. 2017:91(6):962-76.

9. Wang P, Ma L, Wang S, Li L, Wang Q, Yang R, et al. Identification and analysis of a candidate WRKY transcription factor gene affecting adventitious root formation using association mapping in catalpa scop. DNA Cell Biol. 2019;38(4):297-306

10. Huang R, Liu D, Huang M, Ma J, Li Z, Li M, et al. CpWRKY71, a WRKY transcription factor gene of wintersweet (Chimonanthus praecox), promotes flowering and leaf senescence in arabidopsis. Int J Mol Sci. 2019;20(21):5325.
11. Yang $L$, Ye C, Zhao $Y$, Cheng $X$, Wang $Y$, Jiang $Y Q$, et al. An oilseed rape WRKY-type transcription factor regulates ROS accumulation and leaf senescence in Nicotiana benthamiana and Arabidopsis through modulating transcription of RbohD and RbohF. Planta. 2018;247(6):1323-38.

12. Yang $Y$, Wang $N$, Zhao S. Functional characterization of a WRKY family gene involved in somatic embryogenesis in Panax ginseng. Protoplasma. 2020;257(2):449-58.

13. Dai $X$, Wang $Y$, Zhang WH. OsWRKY74, a WRKY transcription factor, modulates tolerance to phosphate starvation in rice. J Exp Bot. 2016;67(3):947-60.

14. Ding ZJ, Yan JY, Xu XY, Yu DQ, Li GX, Zhang SQ, et al. Transcription factor WRKY46 regulates osmotic stress responses and stomatal movement independently in Arabidopsis. Plant J. 2014;79(1):13-27.

15. Yan $\mathrm{H}$, Jia H, Chen $X$, Hao L, An H, Guo X. The cotton WRKY transcription factor GhWRKY17 functions in drought and salt stress in transgenic Nicotiana benthamiana through ABA signaling and the modulation of reactive oxygen species production. Plant Cell Physiol. 2014;55(12):2060-76.

16. Chu X, Wang C, Chen X, Lu W, Li H, Wang X, et al. Correction: The cotton WRKY gene GhWRKY41 positively regulates salt and drought stress tolerance in transgenic nicotiana benthamiana. PLoS One. 2016;11(6):e0157026.

17. He GH, Xu JY, Wang YX, Liu JM, Li PS, Chen M, et al. Drought-responsive WRKY transcription factor genes TaWRKY1 and TaWRKY33 from wheat confer drought and/or heat resistance in Arabidopsis. BMC Plant Biol. 2016;16(1):116.

18. Ding ZJ, Yan JY, Xu XY, Yu DQ, Li GX, Zhang SQ, et al. Transcription factor WRKY 46 regulates osmotic stress responses and stomatal movement independently in A rabidopsis. Plant J. 2014;79(1):13-27.

19. Ong A-L, Teh C-K, Mayes S, Massawe F, Appleton DR, Kulaveerasingam $H$. An improved oil palm genome assembly as a valuable resource for crop improvement and comparative genomics in the arecoideae subfamily. Plants. 2020;9(11):1476.

20. Li X, Tang Y, Zhou C, Zhang L, LV J. A wheat WRKY transcription factor TaWRKY46 enhances tolerance to osmotic stress in transgenic arabidopsis plants. Int J Mol Sci. 2020;21(4):1321.

21. Wei W, Cui MY, Hu Y, Gao K, Xie YG, Jiang Y, et al. Ectopic expression of FVWRKY42, a WRKY transcription factor from the diploid woodland strawberry (Fragaria vesca), enhances resistance to powdery mildew, improves osmotic stress resistance, and increases abscisic acid sensitivity in Arabidopsis. Plant Sci. 2018;275:60-74.

22. Meijaard E, Brooks TM, Carlson KM, Slade EM, Garcia-Ulloa J, Gaveau DLA, et al. The environmental impacts of palm oil in context. Nat Plants. 2020;6(12):1418-26.

23. Omar S, Kamil N. The impact of El Niño and La Niña on Malaysian palm oil industry; 2017.

24. Kamil NN, Omar SF. Climate variability and its impact on the palm oil industry. Oil Palm Indust Econ J. 2016;16(1):18-30.

25. Xiao Y, Zhou L, Lei X, Cao H, Wang Y, Dou Y, et al. Genome-wide identification of WRKY genes and their expression profiles under different abiotic stresses in Elaeis guineensis. PLoS One. 2017;12(12):e0189224.

26. Wen W, Wang R, Su L, Lv A, Zhou P, An Y. MsWRKY11, activated by MsWRKY22, functions in drought tolerance and modulates lignin biosynthesis in alfalfa (Medicago sativa L.). Environ Exp Bot. 2021;184:104373.

27. Kang G, Yan D, Chen X, Yang L, Zeng R. HbWRKY82, a novel Ilc WRKY transcription factor from Hevea brasiliensis associated with abiotic stress tolerance and leaf senescence in Arabidopsis. Physiol Plant. 2021;171(1):151-60.

28. Dang F, Wang Y, She J, Lei Y, Liu Z, Eulgem T, et al. Overexpression of CaWRKY27, a subgroup Ile WRKY transcription factor of Capsicum annuum, positively regulates tobacco resistance to Ralstonia solanacearum infection. J Physiol Plant. 2014;150(3):397-411.

29. Ahammed GJ, Li X, Wan H, Zhou G, Cheng Y. SIWRKY81 reduces drought tolerance by attenuating proline biosynthesis in tomato. Sci Hortic. 2020;270:109444.

30. Chanwala J, Satpati S, Dixit A, Parida A, Giri MK, Dey N. Genome-wide identification and expression analysis of WRKY transcription factors in pearl millet (Pennisetum glaucum) under dehydration and salinity stress. BMC Genomics. 2020;21(1):231. 
31. Wei W, Hu Y, Han YT, Zhang K, Zhao FL, Feng JY. The WRKY transcription factors in the diploid woodland strawberry Fragaria vesca: Identification and expression analysis under biotic and abiotic stresses. Plant Physiol Biochem. 2016;105:129-44.

32. Gao YF, Liu JK, Yang FM, Zhang GY, Wang D, Zhang L, et al. The WRKY transcription factor WRKY 8 promotes resistance to pathogen infection and mediates drought and salt stress tolerance in Solanum lycopersicum. Physiol Plant. 2020;168(1):98-117.

33. Li F, Zhang $L$, Ji H, Xu Z, Zhou Y, Yang S. The specific W-boxes of GAPC5 promoter bound by TaWRKY are involved in drought stress response in wheat. Plant Sci. 2020;296:110460.

34. Li Z, Liang F, Zhang T, Fu N, Pei X, Long Y. Enhanced tolerance to drought stress resulting from caragana korshinskii CkWRKY33 in transgenic Arabidopsis Thaliana. BMC Genomic Data. 2021;22(1):19-10

35. Wang J, Wang L, Yan Y, Zhang S, Li H, Gao Z, et al. GhWRKY 21 regulates ABA-mediated drought tolerance by fine-tuning the expression of GhHAB in cotton. Plant Cell Rep. 2021;40(11):2135-2150.

36. Xiong C, Zhao S, Yu X, Sun Y, Li H, Ruan C, et al. Yellowhorn droughtinduced transcription factor XsWRKY20 acts as a positive regulator in drought stress through ROS homeostasis and ABA signaling pathway. Plant Physiol Biochem. 2020;155:187-95.

37. Yang Z, Chi X, Guo F, Jin X, Luo H, Hawar A, et al. SbWRKY30 enhances the drought tolerance of plants and regulates a drought stress-responsive gene, SbRD19, in sorghum. J Plant Physiol. 2020;246-247:153142.

38. Hao X, Wang S, Chen Y, Qu Y, Yao H, Shen Y. Genome-wide identification and analysis of the WRKY gene family in the Xerophytic Evergreen Ammopiptanthus nanus. Agronomy. 2020;10(11):1634.

39. Yono D, Nugroho YA, Tanjung ZA, Utomo C, Liwang T. Genomewide SNP marker identification associated with drought tolerance in oil palm. Biodivers J Biol Divers. 2021;22(6).

40. Ahmad N, Malagoli M, Wirtz M, Hell R. Drought stress in maize causes differential acclimation responses of glutathione and sulfur metabolism in leaves and roots. BMC Plant Biol. 2016;16(1):247.

41. Miller G, Suzuki N, Ciftci-Yilmaz S, Mittler R. Reactive oxygen species homeostasis and signalling during drought and salinity stresses. Plant Cell Environ. 2010;33(4):453-67.

42. Huang Y, Li M-Y, Wu P, Xu Z-S, Que F, Wang F, et al. Members of WRKY Group III transcription factors are important in TYLCV defense signaling pathway in tomato (Solanum lycopersicum). BMC Genomics. 2016;17(1):1-18.

43. Dou L-L, Guo Y-N, Ondati E, Pang C-Y, Wei H-L, Song M-Z, et al. Identification and expression analysis of group III WRKY transcription factors in cotton. J Integr Agric. 2016;15(11):2469-80.

44. Wang D, Wang L, Su W, Ren Y, You C, Zhang C, et al. A class III WRKY transcription factor in sugarcane was involved in biotic and abiotic stress responses. Sci Rep. 2020;10(1):1-15.

45. Huang Y, Li MY, Wu P, Xu ZS, Que F, Wang F, et al. Members of WRKY Group III transcription factors are important in TYLCV defense signaling pathway in tomato (Solanum lycopersicum). BMC Genomics. 2016;17(1):788.

46. Pieterse CM, Van der Does D, Zamioudis C, Leon-Reyes A, Van Wees, SCJAroc, biology d. Hormonal modulation of plant immunity. Annu Rev Cell Dev Biol. 2012;28:489-521.

47. Wan J, Griffiths R, Ying J, McCourt P, Huang Y. Development of droughttolerant canola (Brassica napus L.) through genetic modulation of ABAmediated stomatal responses. Crop Sci. 2009;49(5):1539-54.

48. Khan N, Bano A, Ali S, Babar MA. Crosstalk amongst phytohormones from planta and PGPR under biotic and abiotic stresses. Plant Growth Regul. 2020;90(2):189-203.

49. Fraire-Velázquez S, Rodríguez-Guerra R, Sánchez-Calderón L. Abiotic and biotic stress response crosstalk in plants. Abiotic stress response in plants_-physiological, biochemical and genetic perspectives; 2011. p. $3-26$.

50. Dang F, Wang Y, She J, Lei Y, Liu Z, Eulgem T, et al. Overexpression of CaWRKY27, a subgroup lle WRKY transcription factor of Capsicum annuum, positively regulates tobacco resistance to Ralstonia solanacearum infection. Physiol Plant. 2014;150(3):397-411.

51. Wang CT, Ru JN, Liu YW, Yang JF, Li M, Xu ZS, et al. The maize WRKY transcription factor ZmWRKY40 confers drought resistance in transgenic arabidopsis. Int J Mol Sci. 2018;19(9):2580.
52. Gill SS, Tuteja N. Reactive oxygen species and antioxidant machinery in abiotic stress tolerance in crop plants. Plant Physiol Biochem. 2010;48(12):909-30.

53. Saibi W, Feki K, Mahmoud RB, Brini F. Durum wheat dehydrin (DHN-5) confers salinity tolerance to transgenic Arabidopsis plants through the regulation of proline metabolism and ROS scavenging system. Planta. 2015;242(5):1187-94.

54. Singh M, Kumar J, Singh S, Singh VP, Prasad SM. Roles of osmoprotectants in improving salinity and drought tolerance in plants: a review. Rev Environ Sci Biotechnol. 2015;14(3):407-26.

55. Xu YH, Sun PW, Tang XL, Gao ZH, Zhang Z, Wei JH. Genome-wide analysis of WRKY transcription factors in Aquilaria sinensis (Lour.) Gilg. Sci Rep. 2020;10(1):3018.

56. Chu X, Wang C, Chen X, Lu W, Li H, Wang X, et al. The cotton WRKY gene GhWRKY41 positively regulates salt and drought stress tolerance in transgenic nicotiana benthamiana. PLoS One. 2015;10(11):e0143022.

57. Kim D, Pertea G, Trapnell C, Pimentel H, Kelley R, Salzberg SL. TopHat2: accurate alignment of transcriptomes in the presence of insertions, deletions and gene fusions. Genome Biol. 2013;14(4):1-13.

58. Anders S, Pyl P, Huber W. HTSeq: analysing high-throughput sequencing data with Python; 2010.

59. Anders S, Huber W. Differential expression of RNA-Seq data at the gene level-the DESeq package, vol. 10. Heidelberg: European Molecular Biology Laboratory; 2012. p. f1000research.

60. Zheng Y, Jiao C, Sun H, Rosli H, Pombo MA, Zhang P, et al. iTAK: a program for genome-wide prediction and classification of plant transcription factors, transcriptional regulators, and protein kinases. Mol Plant. 2016;9(12):16679-1670.

61. Livak KJM, Schmittgen T. Analysis of relative gene expression data using real---time quantitative PCR and the 2---[Delta][Delta] CT method.25; 2001.

62. Yeap W-C, Loo JM, Wong YC, Kulaveerasingam H, Culture O. Evaluation of suitable reference genes for qRT-PCR gene expression normalization in reproductive, vegetative tissues and during fruit development in oil palm. J Plant Cell Tissue. 2014;116(1):55-66.

\section{Publisher's Note}

Springer Nature remains neutral with regard to jurisdictional claims in published maps and institutional affiliations.

Ready to submit your research? Choose BMC and benefit from:

- fast, convenient online submission

- thorough peer review by experienced researchers in your field

- rapid publication on acceptance

- support for research data, including large and complex data types

- gold Open Access which fosters wider collaboration and increased citations

- maximum visibility for your research: over 100M website views per year

At BMC, research is always in progress.

Learn more biomedcentral.com/submissions 